\title{
Tratamento das taquiarritmias durante a gestação
}

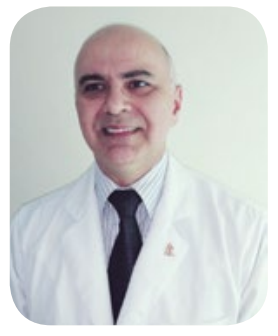

\section{RESUMO}

O organismo da gestante apresenta alterações fisiológicas importantes, que vão interferir na gênese de arritmias e no metabolismo de drogas antiarrítmicas. As limitações no uso de antiarrítmicos por conta de efeitos colaterais maternos e fetais exigem um conhecimento maior da segurança e uso destas drogas. As arritmias, apesar de infrequentes, podem ser muito desconfortáveis no período gestacional. O diagnóstico, a identificação do risco e a instituição de tratamento precoce melhoram a qualidade de vida. Os avanços no tratamento ablativo de arritmias, com exposição mínima à radiação, oferecem tratamento definitivo em casos selecionados durante a gestação.

PALAVRAS-CHAVE: arritmias, gestação, ablação.

\section{TEXTO}

A incidência de arritmias é diferente para homens e mulheres, embora em muitos casos não se saibam os motivos desta diferença. Dois mecanismos têm sido propostos para explicar essas diferenças: efeitos hormonais na função e expressão de canais iônicos, diferenças no tônus autonômico ou a combinação de ambos. ${ }^{1} \mathrm{O}$ que é comprovado na participação feminina, com $70 \%$ dos casos de Torsades de Pointes, induzido por drogas, e em cerca de $20 \%$ de pacientes do sexo feminino, em trials de (MADIT, MADIT II, CABG-PATH, COMPANION, DFINITE, DINAMITE, SCD-HEFT) $)^{1,2,3,4,5,6,7}$ e observações, sobre tratamento ablativo da fibrilação atrial. $8,9,10,11$

A mulher tem ao ECG alterações particulares, como: frequência cardíaca maior, QT corrigido mais longo (maior incidência de QT longo e maior incidência de Torsades de Pointes, porém menos episódios de morte súbita), menor duração e voltagem do QRS. ${ }^{1}$

\author{
Bráulio Pinna $^{1}$
}

A gestação apresenta alterações fisiológicas que podem criar meio propício à arritmogênese: 1- Alterações do estado hormonal (aumento do estrogênio e do B-HCG), que podem alterar a ação dos canais iônicos; 2- Alterações hemodinâmicas - $o$ aumento do volume circulante e consequente aumento do débito cardíaco, levando à dilatação miocárdica e ao aumento do volume diastólico final; 3- Aumento do tônus simpático causado por altas concentrações plasmáticas de catecolaminas e sensibilidade do receptor adrenérgico.
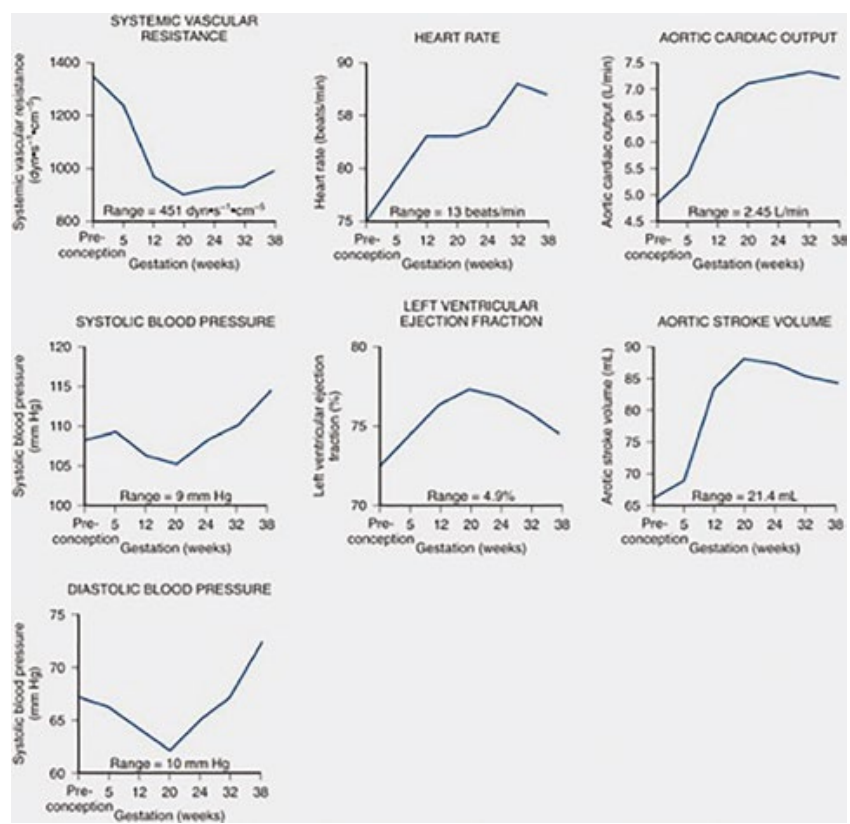

Figura 1 - Alterações hemodinâmicas e circulatórias em mulheres com gestação normal ${ }^{12}$. 


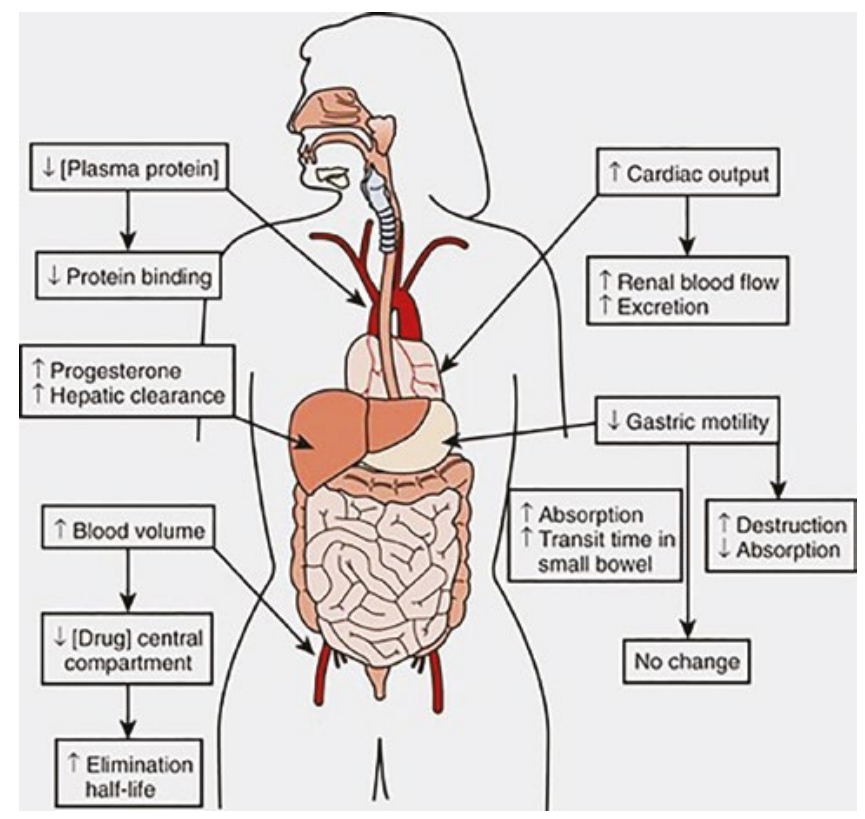

Figura 2 - Alterações de absorção, distribuição e eliminação de drogas farmacológicas no organismo da gestante normal' ${ }^{12}$.

Para a administração e prescrição de medicações durante a gestação, deve-se levar em consideração: a farmacocinética, avaliando e necessidade de ajuste da dose da droga ou se há a possibilidade de monitoração de valores séricos ou efeitos colaterais. Pelos riscos de teratogenicidade, devemos avaliar a descontinuação ou substituição por outra droga e dosar risco-benefício para a mãe e para o feto. O maior de todos os entraves é a aceitação pela mãe de fazer uso da medicação, por conta da fragilidade emocional e o instinto de proteção do feto. ${ }^{13}$

Em observação publicada por Li et al., 136.428 gestantes foram acompanhadas pelo período de 8 anos e as arritmias mais frequentes foram $60 \%$ de arritmias sinusais (bradicardias e taquicardias), seguidas por $19 \%$ de extrassístoles atriais e ventriculares, $14 \%$ de taquicardias supraventriculares e, mais raras, $1 \%$ de fibrilação atrial e $1 \%$ de taquicardias ventriculares. ${ }^{14}$

O uso de drogas antiarrítmicas está relacionado com a idade gestacional, o risco e sintomas maternos. Segundo o FDA, não há droga antiarrítmica com grau de segurança A. O Sotalol (nível de segurança B) é uma droga segura para tratamento materno, podendo ser usada para tratamento de arritmias fetais (nível de segurança B), seguido por Metoprolol, Propranolol, Propafenona, Digoxina e Adenosina (nível de segurança C). A Amiodarona e o Atenolol devem ser evitados em qualquer período da gestação. ${ }^{15,16,17,18,19,21}$ (Figura 3)

\begin{tabular}{|c|c|c|c|c|c|c|}
\hline Drug & $\begin{array}{l}\text { Drug } \\
\text { class" }\end{array}$ & $\begin{array}{l}\text { FDA } \\
\text { closs }\end{array}$ & Potential adverse effects & Primary indication[s] & Use during lactation" & Comments \\
\hline Quinidire & IA & $C$ & 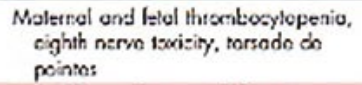 & $\begin{array}{l}\text { Votisly of moletnol and felal } \\
\text { orrhythmias }\end{array}$ & $\begin{array}{l}\text { Genetally compafible bul } \\
\text { coution otrised }\end{array}$ & Long resord of salsly \\
\hline Procainemido & IA & c & $\begin{array}{l}\text { tupus like syndromo with longserm } \\
\text { use, torsode de pointes }\end{array}$ & $\begin{array}{l}\text { Drug of choice fr } \propto \text { uto troctmont } \\
\text { cl undiagnosed wide complex } \\
\text { tochycordio }\end{array}$ & $\begin{array}{l}\text { Compatibls but longterm } \\
\text { therapy showld be } \\
\text { avided }\end{array}$ & $\begin{array}{l}\text { Has the advantage of intravanous } \\
\text { desing, long record of sdely }\end{array}$ \\
\hline Discpyromise & IA & $\mathrm{C}$ & $\begin{array}{l}\text { Indyetion of ulerine controctiens. } \\
\text { Isrsods de pcinlas }\end{array}$ & $\begin{array}{l}\text { Limiled expetience, oher } \\
\text { cltarnative: availabls }\end{array}$ & Compatible & Limited experiense \\
\hline Lidocaine & 18 & B & CNS odverse effects, bradyeardia & $\begin{array}{l}\text { Natemal ventrisular tachycardia, } \\
\text { orrhythmias duo } 10 \text { digoxin loxicity }\end{array}$ & Compatible & $\begin{array}{l}\text { Leng resond of safkly, aveid if fetal } \\
\text { distress }\end{array}$ \\
\hline Nexielin: & 18 & $C$ & $\begin{array}{l}\text { CNS odveron éliscls, latal } \\
\text { trodyecordio, low APGAR. }\end{array}$ & Ventricubr tochycordio & Compatible & Limited expetiense \\
\hline Phemysin & IB & D & $\begin{array}{l}\text { Mental and gresth retardation, fotel } \\
\text { hydenloin syndtome }\end{array}$ & Arrhythmios due to digoxin toxicity & $\begin{array}{l}\text { Genorally compotibla bet } \\
\text { coution ostrived }\end{array}$ & $\begin{array}{l}\text { Bemer alernotives availablo, avoid if } \\
\text { possible }\end{array}$ \\
\hline Flecoinide & IC & $C$ & $\begin{array}{l}\text { Insteosed moriality in palients with } \\
\text { pevious myecuidiel inlarclion. } \\
\text { generally woll islerased in } \\
\text { strueverally normd heorts }\end{array}$ & $\begin{array}{l}\text { Vorisly of malemol and febl } \\
\text { ventricular tochycardio ond SVT }\end{array}$ & Compstible & $\begin{array}{l}\text { First line cption for lieoling lebl SVT } \\
\text { with hydeps }\end{array}$ \\
\hline Propdenono & IC & C & $\begin{array}{l}\text { Samo concorns as fieccinido, mild } \\
\text { B-blecket effects }\end{array}$ & $\begin{array}{l}\text { Variety of maternal ventrisular } \\
\text { tochycordio and SVT }\end{array}$ & Unknown & Limitod experiense \\
\hline $\begin{array}{l}\text { Bblocking } \\
\text { agents }\end{array}$ & ॥ & $\mathrm{C} / \mathrm{D}$ & $\begin{array}{l}\text { Introuterine growth retordotion, fetol } \\
\text { trodycardio, hypoglycemio, fesol } \\
\text { qonea }\end{array}$ & $\begin{array}{l}\text { Motemol SVT, idispathic wentriculor } \\
\text { sochycordio, arial fibrillotion rote } \\
\text { centrd }\end{array}$ & $\begin{array}{l}\text { Avoid alenokd. Metoprolal } \\
\text { and propronold are } \\
\text { compatible }\end{array}$ & $\begin{array}{l}\text { Generolly well roleroted, ovoid } \\
\text { during first trimaster, cordoc } \\
\text { selective ore prelened }\end{array}$ \\
\hline Arrioderene & III & D & $\begin{array}{l}\text { Feld hypcthyroidiam, premolurity, } \\
\text { low bitth weight, congenild } \\
\text { moliformations }\end{array}$ & $\begin{array}{l}\text { Life-threatening ventricular } \\
\text { crithythmio: }\end{array}$ & $\begin{array}{l}\text { Avoid since a laige } \\
\text { amcent of diug is } \\
\text { obcorbod by the infant }\end{array}$ & $\begin{array}{l}\text { Avoid if possible especially during } \\
\text { firtl trimesler }\end{array}$ \\
\hline Srablel & III & B & Torsods do pointos, f-blocker effects & $\begin{array}{l}\text { Motomal ventriziar tachyeardia and } \\
\text { SVT, hypertonsion in the post }\end{array}$ & $\begin{array}{l}\text { Generally compotiblo bet } \\
\text { coution otrised }\end{array}$ & limisod axporiense \\
\hline Ibutlids & IIII & c & Torsode de pointas & $\begin{array}{l}\text { Acuto mormination of atrial fibrillotion } \\
\text { /lluller }\end{array}$ & Unknown & No exporienco in pregnancy \\
\hline
\end{tabular}




\begin{tabular}{|c|c|c|c|c|c|c|}
\hline Drenedarone & III & $x$ & Torsode de pointe: & Astial fibrillation & Contraindicated & Controindiculed \\
\hline Dolestide & III & C & Torsode de pointes & $\begin{array}{l}\text { Arial fibrillation in the selling of } \\
\text { stuctural heorl diseose }\end{array}$ & Unknown & No experience in pregnancy \\
\hline \multicolumn{7}{|c|}{ Table 2 (Confinued) } \\
\hline Drug & $\begin{array}{l}\text { Drug } \\
\text { class" }\end{array}$ & $\begin{array}{l}\text { FDA } \\
\text { class" }\end{array}$ & Potential adveree effects & Primary indication(s) & Use during loctation" & Comments \\
\hline Veropamil & Iy & C & $\begin{array}{l}\text { Maternal hypedension, Etal } \\
\text { krodyeordio and hoon black }\end{array}$ & $\begin{array}{l}\text { Maternd and letal SVT, idispathis } \\
\text { vontricular sochycordio, otriol } \\
\text { fbrillotion roto control }\end{array}$ & Compatible & $\begin{array}{l}\text { Reletively well tolerated bel sefer } \\
\text { options aro oveiloblo }\end{array}$ \\
\hline Difiazem & I & C & $\begin{array}{l}\text { Limiled experience, same concerns as } \\
\text { with vercpomil }\end{array}$ & $\begin{array}{l}\text { Molemd SVT, oltial fibrillotion role } \\
\text { control }\end{array}$ & Compatible & $\begin{array}{l}\text { Vercpomil preferred due to bonger } \\
\text { rocord in pregnancy }\end{array}$ \\
\hline Adenosine & $N / A$ & C & Dyspnea, brodycordia & Acute lermination of malernol SVI & $\begin{array}{l}\text { Unknown, but probably } \\
\text { well nolorotod }\end{array}$ & First option for ocute treatment of SVT \\
\hline Digoxin & $N / A$ & c & Low binh weight & $\begin{array}{l}\text { Fetal and malernal SVT, atrial fibrillo } \\
\text { tion rate control }\end{array}$ & Compatible & Long record of salety \\
\hline
\end{tabular}

Figura 3 - Drogas antiarrítmicas e o seu risco durante a gestação ${ }^{16}$.

\section{Taquicardias Supraventriculares (TPSV)}

Gestantes com ritmo sinusal de base apresentam $44 \%$ de chance de recorrência da taquiarritmia, $34 \%$ de chance de novo episódio de TPSV e até $29 \%$ apresentam exacerbação da arritmia no episódio de TPSV 21, 22, 23. Para o tratamento agudo dessas arritmias, estão indicadas as mesmas orientações especificadas para pacientes não gestantes: manobra vagal, adenosina e/ou cardioversão elétrica; Metoprolol intravenoso ou até o Verapamil intravenoso podem ser utilizados. Para manutenção do ritmo sinusal em períodos inter-críticos (evitar recorrência), drogas como o Sotalol, Metoprolol e outras podem ser utilizadas, porém em pacientes muito sintomáticas e com crises frequentes de TPSV. ${ }^{16-18,21}$

\section{Fibrilação e flutter atrial}

São arritmias extremamente raras durante a gestação e sua ocorrência é mais frequente (até 96\%) em pacientes portadoras de comorbidades, como: doença valvular reumática, cardiopatias congênitas ou hipertireoidismo. ${ }^{21} \mathrm{~A}$ manutenção do ritmo sinusal pode ser feita com Sotalol ou Flecainida e, no caso de necessidade de reversão aguda, a cardioversão elétrica está indicada. A Amiodarona deve ser evitada e restrita à emergência (se não houver sucesso de reversão do ritmo com as medidas citadas), pelo alto risco de teratogenicidade. ${ }^{21,24,25}$ Para 0 controle da frequência cardíaca recomenda-se, em primeira linha, o uso de b-bloqueador e/ou digoxina, como segunda linha, o Verapamil ou Diltiazen podem ser utilizados. ${ }^{21,24,25}$

\section{Taquicardia Ventricular (TV)}

A presença de alterações fisiológicas hiperdinâmi- cas da gestação pode desencadear TV Catecolaminérgica ou Idiopática de VD, estas estão entre as mais frequentes dentro do universo raro das TVs. As alterações estruturais miocárdicas, como miocardiopatia periparto, miocardiopatia hipertrófica, doença arterial coronária, cardiopatia reumática e outras, podem apresentar raros episódios de TV ${ }^{26}$. Portadoras de síndrome do QT Longo têm risco aumentado de desenvolver TV no puerpério. Portanto, o uso de b-bloqueador deve ser mantido após a gestação e o parto. Mulheres com Síndrome de Brugada não apresentam grandes riscos de arritmias ventriculares complexas durante a gestação. ${ }^{26}$

No diagnóstico de TV, principalmente se houver repercussão hemodinâmica, a cardioversão elétrica é a primeira opção de tratamento. A indicação de cardiodesfibrilador implantável se faz necessária quando a TV e a consequente indicação do implante ocorre durante a gestação. Nos casos de Síndrome do QT Longo ou TV idiopática de via de saída de ventrículo direito, está indicado o uso de b-bloqueador (Metoprolol ou Propranolol). ${ }^{26}$

$O$ risco de arritmias, de qualquer gênese, como complicação cardiovascular em gestantes é de cerca de $26 \%$ em pacientes que, na sua maioria, são portadoras de valvopatia reumática ${ }^{27}$.

\section{Tratamento por radiofrequência}

A ablação por cateter é um método eficaz para tratamento definitivo de muitas arritmias e pode oferecer cura, com baixo risco de complicações, em pacientes em geral. A indicação nas pacientes gestantes somente deve ser feita nos casos de intolerância à medicação, falência do tratamento farmacológico, crises de repetição e mal 
toleradas pela paciente. $O$ grande risco é a exposição do feto à radiação emitida durante a fluoroscopia, por este motivo é recomendada a realização deste procedimento, utilizando o sistema de navegação com mapeamento eletroanatômico, que reduz a exposição à radiação a tempo mínimo ou até mesmo a realização sem radiação. ${ }^{23,28}$

TABLE: 1

Characteristics of Individual Patients from Included Reports

\begin{tabular}{|c|c|c|c|c|c|c|}
\hline $\begin{array}{l}\text { Author } \\
\text { and Date }\end{array}$ & Presentation & $\begin{array}{l}\text { Tachycardia } \\
\text { Mechanism }\end{array}$ & $\begin{array}{c}\text { Malermal } \\
\text { Age (Years) }\end{array}$ & $\begin{array}{l}\text { (iexlation } \\
\text { (Weeks) }\end{array}$ & $\begin{array}{l}\text { Plumrousopy I Imu's } \\
\text { Dose (Seconds) }\end{array}$ & M:spping \\
\hline Grax 13 & Rexument palpitalium after cardiumersios & AFI. AF & 27 & 22 & NR & $N_{\text {II }}$ \\
\hline Dominguez. ${ }^{12}$ & Recument SVT & ORT. R AP & 31 & 20 & $70\langle\mathrm{NR}\rangle$ & No \\
\hline \multirow[t]{3}{*}{ Bombelli: } & Rocument SVT, drug refractory & AVNRT & 28 & 30 & $516(1.5 \mathrm{mGy})$ & No \\
\hline & Recurrent SVT. druy, refractory & ORT. IA. AP & 27 & 29 & $408(1.2 \mathrm{mG})$ & No \\
\hline & Recument SIT. drug refractory & ORT. R AP & 32 & 30 & $1.776(5.2 \mathrm{mG} \dot{y})$ & No \\
\hline Pagad ${ }^{16}$ & Congestive heart fallure ( $\mathrm{EF} 22(\tilde{c})$ & $\mathrm{R} \wedge \mathrm{T}$ & 24 & 26 & $240(\mathrm{NR})$ & No \\
\hline Kanjwal ${ }^{24}$ & Presynoxyx, jailpitation, inug retractury & ORT,I」. AP & 32 & 22 & 457 (NR) & Niı \\
\hline \multirow[t]{2}{*}{ Berruesos 9} & Palpitations (TF" -30\%) & R AT (RAA) & 30 & 30 & $481(0.25 \mathrm{mGy})$ & No \\
\hline & $\mathrm{NR}$ & ORT. LL AP & 22 & 12 & $109(0.1 \mathrm{mGy})$ & No \\
\hline Bongiomill & Palpitations & AVNKI & 32 & 10 & 0 & $\mathrm{ICE}$ \\
\hline \multirow[t]{9}{*}{ Szumowskis } & Palpitations (EF 45\%) & R AT & $3 !$ & 24 & 0 & Yes. TTE \\
\hline & Recument syncope (EF $41 \%)$ & $\mathrm{R} \wedge \mathrm{I}$ & 29 & 37 & 70 (NR) & CARTO \\
\hline & Csxugesliwe heat failume (F.F $2\left(K K_{\tilde{S}}\right)$ & PJRT & 24 & 21 & (1) & Yex \\
\hline & I.N dysurction (T:F 41 for) & PJRT & 27 & 33 & 0 & Yex \\
\hline & Drug refractory & PJRT & 31 & 38 & $28(\mathrm{NR})$ & Yes \\
\hline & Drug refrastory, recument after cardioversion & AVNRT & 24 & 35 & 53 (NR) & No \\
\hline & Presynocpe. fetal decelerations & ORT. LL AP & $3 !$ & $3 !$ & $106(\mathrm{NR})$ & No \\
\hline & Syncope & ORT, LL AP & 27 & 22 & $57(\mathrm{NR})$ & No \\
\hline & Syncope & ORI, L AP & 27 & 12 & $\theta(N K)$ & $\mathrm{Nn}$ \\
\hline Fergusm 10 & Coxugestive hest failure (ГT. $40 / \hat{x})$ & I. AT & 20 & 27 & 0 & NavXICT: \\
\hline Mianjaly ${ }^{15}$ & VF (preexcited AF) & ORT. R AP (CS OS) & 33 & 15 & 0 & NavX \\
\hline $\mathrm{Wu}^{13}$ & Symptomatic palpitations and dyspnsa (EF $40 K_{0}$ ) & $\mathrm{R} \wedge \mathrm{T}(\mathrm{R} \Lambda \lambda)$ & 32 & 14 & $55(<0.1 \mathrm{mGy})$ & NavX \\
\hline Stecs & VT (anhythmogenic RV cardiomyurathy) & $\mathrm{KV} \mathrm{VI}$ & 26 & 23 & (1) (0.1 m(iy) & (AARTO \\
\hline Clark: $^{18}$ & Rocument SVT, drug refractory & AVNRT & 27 & 22 & 0 & NavX \\
\hline Hogarth ${ }^{5}$ & VI, presyncope, palpitations, drug refractory & RVCI'V'I' & 36 & 31 & (1) $(9.9 \mathrm{~m}$ (iy) & NavX \\
\hline I.ciris ${ }^{19}$ & Symoipe & ORT.IJ. AP & 33 & 26 & 0 & NavX \\
\hline Zuberi ${ }^{3)}$ & Congestive heart failure (EF $30^{\prime}{ }^{\prime} c$ ) & R AT & 48 & 30 & 0 & NavX \\
\hline
\end{tabular}

Rediation dosing was variously reportod in milligray ( $\mathrm{m}(\mathrm{jy})$ and mrad, converted here to $\mathrm{mGy}: \mathrm{NR}=\mathrm{n} \propto$ reported.

Figura 4 - Tempo de exposição à radiação em pacientes submetidos à ablação guiada por fluoroscopia e mapeamento eletroanatômico. ${ }^{29}$

Kevin et al. demonstram a diminuição de exposição à radiação, o que é confirmado por Chen et al. com a publicação de dois casos de ablação realizados com mapeamento eletroanatômico com sucesso e sem complicações e apresenta revisão de publicações onde se nota a segurança deste método para pacientes gestantes, observando o pequeno número de procedimentos realizados e a escolha de submeter essas pacientes ao procedimento ablativo após 0 segundo ${ }^{27}$ (Figuras 5 e 6 ).

\section{Table 2}

Results of the procedures and the follow up.

\begin{tabular}{lcc}
\hline & Patient 1 & Patient 2 \\
\hline Procedure time, min & 41 & 71 \\
Complications & No & No \\
Follow up, mo & 24 & 6 \\
New born weight, $g$ & 3050 & 2700 and 1920 \\
Weeks of delivery, HbD & 38 & 35 \\
Type of delivery & CC & CC \\
\hline
\end{tabular}

$\mathrm{CC}=\mathrm{C}$ esarean section, $\mathrm{HbD}=$ weeks of delivery.

Figura 5 - Resultados pós-ablação guiada por mapeamento eletroanatômico, relato de dois casos $^{22}$. 
Tables

Characteristics of pregnant paticnts from reported cascs and our cascs.

\begin{tabular}{|c|c|c|c|c|c|c|}
\hline Author & Presentation & Tachycardia mechanism & Maternal age (years old) & Gestation (weeks) & Mapping & Rets. \\
\hline Bangioml $(2006)$ & Palditations & AVIRT & 32 & 10 & ICE & [10] \\
\hline \multirow{3}{*}{ Srumowsid (2010) } & Papltations (EF 45\%) & AT & 31 & 24 & TTE & {$[17]$} \\
\hline & Heent faiure (EF 20\%) & PJRT & 24 & 21 & Yes & {$[17]$} \\
\hline & Hean faiure (EF 41\%) & PJAT & 27 & 33 & Yes & {$[1]$} \\
\hline Ferguson (2011) & Heent faiure (EF 40\%) & AT & 20 & 27 & Na:XNAE & {$[16]$} \\
\hline Manja/ (2011) & VF (preeccled Af) & AVRT & 33 & 15 & NavX. & {$[12]$} \\
\hline Leir's (2014) & Sycope & AVRT & 33 & 26 & Navix. & {$[x]$} \\
\hline Zuberi (2014) & Heent faiure (EF OD\%) & AT & 48 & 30 & Navi. & [3] \\
\hline \multirow[t]{3}{*}{ Omaygenc (2015) } & Palpitatons & AVRT & 27 & 21 & Navix. & $m$ \\
\hline & $N \alpha$ seported & AVNRT & 21 & 30 & Navx & {$[2]$} \\
\hline & Nar-syncope & AVRT & 25 & 12 & Navix. & {$[2]$} \\
\hline Bigdar (2015) & Papitations & AVMRT & 27 & 22 & Navix. & $\mu !$ \\
\hline Case 1 & Palpitation & PVCNT & 33 & 31 & $\mathrm{Na} \times \mathrm{X}$ & \\
\hline Case 2 & Palpitations & AVRT & 22 & 25 & NavX & \\
\hline
\end{tabular}

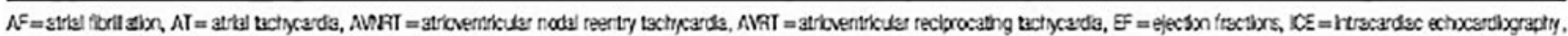

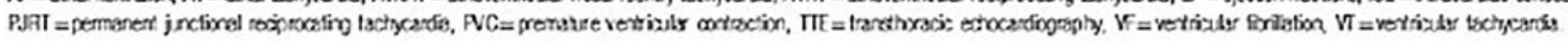

Figura 6 - Características maternas, idade e idade gestacional de pacientes submetidas à ablação²2.

\section{Anticoagulação}

A presença da fibrilação atrial, quando há risco de acidente vascular cerebral, indica o uso de anticoagulação oral. Durante a gestação é recomendado o uso de heparina com dose ajustada, durante o primeiro trimestre e 2 a 4 semanas antes da data prevista do parto. Os antagonistas de vitamina $\mathrm{K}$ podem ser prescritos no período restante gestacional. ${ }^{24}$

Os cumarínicos, apesar de autorizados na gestação, podem causar embriopatia varfarínica, caracterizada por lesões esqueléticas em $5 \%$ dos recém-natos expostos a cumarínicos, entre a $6^{\mathrm{a}}$ e a $12^{\mathrm{a}}$ semana de gestação. ${ }^{25}$

$\mathrm{Na}$ valvopatia reumática e/ou uso de próteses metálicas, o uso de anticoagulação oral se faz necessário. Há recomendações de descontinuação do cumarínico e substituição por heparina de baixo peso molecular, no período compreendido entre a $6^{\mathrm{a}}$ e a $12^{\mathrm{a}}$ semana. ${ }^{30}$

O uso de novos anticoagulantes orais está contra-indicado na gestação e/ou em mulheres que estejam em programação de gestação, por conta do desconhecimento do comportamento desta droga neste grupo de pacientes. ${ }^{24}$

\section{CONCLUSÃO}

Qualquer decisão para escolha do tratamento e da terapêutica para arritmias em pacientes gestantes, pelos riscos pertinentes, deve ser tomada em regime multidisciplinar (arritmologista, cardiologista, obstetra, familiares). Porém, a decisão final será dada pela própria paciente.

\section{REFERÊNCIAS}

1- Oscar Bernal and Concepción Moro. Cardiac Arrhythmias in Women. RevEspCardiol. 2006;59(6):609-18.

2- Moss AJ, Hall WJ, Cannom DS, Daubert JP, Higgins SL, Klein $\mathrm{H}$, et al. Improved survival with na implanted defibrillator in patients with coronary heart disease at high risk of ventricular arrhythmia. N Engl J Med. 1996;335:1933-40.

3- Moss AJ, Zareba W, Hall WJ, Klein H, Wilber D, Cannom DS, et al. The multicenter automatic defibrillator implantation trial II investigators. Prophylactic implantation of a defibrillator in patients with myocardial infarction and reduced ejection fraction. N Engl J Med. 2002;346:877-83.

4- Bigger JT. Prophylactic use of implanted cardiac defibrillators in patientsat high risk of ventricular arrhythmias after coronary artery graft surgery. CABG Patch Trial investigators. N Engl J Med. 1997;337:1569-75.

5- Bristow MR, Saxon LA, Boehmer J, Krueger $\mathrm{S}$, Kass DA, de Marco T, et al. Comparison of medical therapy, pacingand defibrillation in advanced chronic heart failure COMPANION investigators. Cardiac resynchronization the rapy with or without implantable defibrillator in advanced chronic heart failure. N Engl J Med. 2004;350:2140-50.

6- Kadish A, Dyer A, Daubert J, Quigg R, Estes $\mathrm{NA}$, Anderson K, et al. Defibrillators in non-ischemic cardiomyopathy treatment evaluation (DEFINITE) investigators. Prophylactic implantation in patients 
with non-ischemic dilated cardiomyopathy. $\mathrm{N}$ Engl J Med. 2004;350:2151-8.

7- Hohnloser SH, Kuck H, Dorian P, Roberts RS, Hampton JR, Hatala R, et al, the DINAMIT investigators. Prophylactic use of na implantable cardioverter-defibrillator after acute myocardial infarction. N Engl J Med. 2004;351:2481-8.

8- Bardy GH, Lee KL, Mark DB, Poole JE, Packer DL, Boineau R, et al. The sudden cardiac death in heart failure trial (SCD HeFT) investigators. Amiodarone or na implantable cardioverter defibrillator for congestive heart failure. $\mathrm{N}$ Engl J Med. 2005;352:225-37.

9- Haissaguerre M, Jais P, Sha DC, Takahasi A, Hocini M, Quiniou G, et al. Spontaneous initiation of atrial fibrillation by ectopic beats originating in the pulmonary veins. N Engl J Med. 1998;339:659-66.

10- Hocini M, Jais P, Sanders P, Takahasi A, Rotter M, Rostock Th, et al. Techniques, evaluation and consequences of linear block at the left atrial roof in paroxysmal atrial fibrillation. A prospective and randomized study. Circulation. 2005;112:3688-96.

11- Haissaguerre M, Sanders, Hocini M, Takahasi $A$, Rotter M, Sacher F, et al. Catheter ablation of long lasting persistent atrial fibrillation. J Cardiovasc Electro physiol. 2005;16:1125-37.

12- Oral H, Pappone C, Chugh A, Good E, Bogun $F$, Pelosi $F$, et al. Circumferential pulmonary vein ablation for chronic atrial fibrillation. $N$ Engl $J$ Med. 2006;354:934-41.

13- Burkart, Thomas Adam; Miles, William M.; Conti, Jamie Beth. Principles of Arrhythmia Management During Pregnancy. Cviajournal. February 19, 2016 Reviews,Volume 1 Number 2

14- Pieper PG. Use of medication for cardiovascular disease during pregnancy.Nat RevCardiol. 2015 Dec;12(12):718-29.

15- Jian-Ming Li, Carol Nguyen, Jose A. Joglar, Mohamed H. Hamdan, Richard L Page. Frequency and Outcome of Arrhythmias Complicating Admission During Pregnancy: Experience From a High-volume and Ethnically-diverse Obstetric Service. Clin. Cardiol. 200831, 11, 538-541

16- Jose A. Joglar, Richard L. Page. Management of arrhythmia syndromes during Pregnancy. CurrOpinCardiol 2014, 29:36-44

17- Vera Regitz-Zagrosek, et al. ESC Guidelines on the management of cardiovascular diseases during pregnancy. European Heart Journal (2011) 32, 3147-3197

18-Jonathan L. Halperin, et al. ACC/AHA/HRS
Guideline for the Management of Adult Patients With Supraventricular Tachycardia. Circulation. 2016;133:e506-e574

19- Scott L. Ciarkowski, Caren M, Stalburg. Medication Safety in Obstetrics and Gynecology. CLINICAL OBSTETRICS AND GYNECOLOGY. Volume 53, Number 3, 482-499

20- L. B. Van DerHeijden, M.. A. Oudijk, G. T. R. Manten, H. TerHeide, L. Pistorius, M. W. Freund. Sotalol as first-line treatment for fetal tachycardia and neonatal follow-up. Ultrasound Obstet Gynecol 2013; 42: 285-293

21- Alan D. Enriquez, Katherine E. Economy, Usha B. Tedrow. Contemporary Management of Arrhythmias During Pregnancy. Circ Arrhythm Electrophysiol. 2014;7:961-967

22- Lee Shih-Huang, Chen Shih-Ann, Wu Tsu-Juey, Chiang Chern-En, Cheng Chen-Chuen, Tai Ching-Tai, Chiou Chuen-Wang, Ueng Kwo-Chang, Chang Mau-Song. Effects of pregnancy on first on set and symptoms of paroxysmal supraventricular tachycardia. Am J Card. October 1, 1995; Volume 76, Issue 10, Pages 675-678

23- Candice K. Silversides, Louise Harris, Kym Haberer, MathewSermer, Jack M. Colman,Samuel C. Siu. Recurrence Rates of Arrhythmias During Pregnancy in Women With Previous Tachyarrhythmia and Impacton Fetal and Neonatal Outcomes. Am J Cardiol 2006;97:1206 -1212.

24- Paulus Kirchhof, et al. ESC Guidelines for the management of atrial fibrillation developed in collaboration with EACTS. Eur Heart Journal;October 3, 2016

25- Adalberto Lorga Filho, et al. Diretrizes Brasileiras de Fibrilação Atrial. ArqBrasCardiol.2009;92(6 supl.1):1-39

26- Silvia G. Priori. et al.,ESC Guidelines for the management of patients with ventricular arrhythmias and the prevention of sudden cardiac death. European Heart Journal (2015) 36, 2793-2867.

27- Luciana Carvalho Martins, Cláudia Maria Vilas Freire, Carolina Andrade Bragança Capuruçu, Maria do Carmo Pereira Nunes, Cezar Alencar de Lima Rezende. Predição de Risco de Complicações Cardiovasculares em Gestantes Portadoras de Cardiopatia. Arq. Bras. Cardiol. 2016; 106(4):289-296.

28- Guangzhi Chen, et al. Zero-fluoroscopy catheter ablation of severe drug-resistant arrhythmia guided by Ensite Nav Xsystem during pregnancy. Medicine (2016) 95:32(e4487)

29- Kevin Driver, Christian A. Chisholm, Andrew 
E. Darby, Rohit Malhotra, John P. Dimarco, John D. Ferguson. Catheter Ablation of Arrhythmia During Pregnancy. J Cardiovasc Electrophysiol, Vol.26, pp. 698-702, June2015

30- Claire McLintock. Anticoagulant choices in pregnant women with mechanical heart valves: $\mathrm{Ba}-$ lancing maternal and fetal risks - the difference the dose makes. Thrombosis Research 131, Suppl. 1 (2013) S8-S10

1- Serviço de Cardiologia Clínica do HSI Endereço para correspondência:

pinnajunior@uol.com.br 